\title{
Coarse and Surrogate Model Assessment for Engineering Design Optimization with Space Mapping
}

\author{
Slawomir Koziel, Member, IEEE, and John W. Bandler, Life Fellow, IEEE \\ Department of Electrical and Computer Eng., McMaster University, Hamilton, ON, Canada L8S 4K1
}

\begin{abstract}
Two important issues in space mapping optimization are: (i) the quality of the coarse model used in the optimization process, and (ii) the right choice of the space mapping surrogate model for a given optimization problem. Both issues are critical to the performance of the space mapping algorithm. In this paper we introduce methods of assessing the quality of coarse/surrogate models. The methods can be used to predict whether a given combination of a coarse model and a space mapping type can be successfully used in space mapping optimization. They also allow us to compare different surrogate models with respect to potential performance in optimization. Theoretical considerations are illustrated by examples.
\end{abstract}

Index Terms-Computer-aided design(CAD), EM optimization, surrogate model assessment, space mapping.

\section{INTRODUCTION}

Space mapping (SM) has been widely used for optimization and modeling of microwave devices and structures [1]-[5]. SM shifts the optimization burden from an expensive "fine" (or high-fidelity) model to a cheap "coarse" (or low-fidelity) model by iterative optimization and updating of the surrogate model which is built using the coarse model and available fine model data.

The two central issues in space mapping optimization are the quality of the coarse model and the choice of the space mapping surrogate model for a given problem. Both may be critical to the performance of the space mapping algorithm. The coarse model should be as accurate a representation of the fine model as possible. However, simple visual examination of the similarity of the coarse and fine model responses is typically not sufficient to predict the performance of a given coarse model in the space mapping algorithm.

On the other hand, various types of space mapping such as input SM [4], implicit SM [2], output SM [4], frequency SM [3], manifold mapping [6], etc., can be combined in different configurations [4], [5]. Although it seems clear that combining different kinds of space mapping and introducing

This work was supported in part by the Natural Sciences and Engineering Research Council of Canada under Grants OGP0007239 and STPGP336760, and by Bandler Corporation.

S. Koziel is with the Simulation Optimization Systems Research Laboratory, Department of Electrical and Computer Engineering, McMaster University, Hamilton, ON, Canada L8S 4K1.

J.W. Bandler is with the Simulation Optimization Systems Research Laboratory, Department of Electrical and Computer Engineering, McMaster University, Hamilton, ON, Canada L8S 4K1 and also with Bandler Corporation, Dundas, ON, Canada L9H 5E7. new parameters improves the flexibility of the surrogate model, the proper choice of space mapping is usually problem dependent. We do not want the surrogate model to be too simple, because in that case it cannot properly reflect the features of the fine model. Also, we do not want the surrogate to be over-flexible, because its generalization properties may be then lost. In general, a suitable choice of space mapping requires both knowledge of the problem and engineering experience.

A wrong choice of the coarse model and/or space mapping type may result in poor performance of the SM algorithm and significant increase of the optimization cost because a typical approach is to try different space mapping combinations one after another.

In this paper we provide methods of assessing the coarse and surrogate model, which allow predicting the performance of a given combination of a coarse model and space mapping type before carrying out actual space mapping optimization. The methods can be used to select the coarse model and the mapping which are the best for a given optimization problem.

\section{SPACE MAPPING OPTIMIZATION}

Let $R_{f}: X_{f} \rightarrow R^{m}, X_{f} \subseteq R^{n}$, denote the response vector of a fine model of the device of interest. Our goal is to solve

$$
\boldsymbol{x}_{f}^{*}=\arg \min _{x \in X_{f}} U\left(R_{f}(\boldsymbol{x})\right)
$$

where $U: R^{m} \rightarrow R$ is a given objective function. We consider an optimization algorithm that generates a sequence of points $\boldsymbol{x}^{(i)} \in X_{f}, i=0,1,2, \ldots$, and a family of surrogate models $R_{s}^{(i)}: X_{s}^{(i)} \rightarrow R^{m}, X_{s}^{(i)} \subseteq R^{n}, i=0,1, \ldots$, so that

$$
\boldsymbol{x}^{(i+1)}=\arg \min _{\boldsymbol{x} \in X_{f} \cap X_{s}^{(i)}} U\left(\boldsymbol{R}_{s}^{(i)}(\boldsymbol{x})\right)
$$

and $R_{s}^{(i+1)}$ is constructed using suitable matching conditions with the fine model at $\boldsymbol{x}^{(k)}, k=0,1, \ldots, i$.

Let $R_{c}: X_{c} \rightarrow R^{m}, X_{c} \subseteq R^{n}$, denote the response vectors of the coarse model that describes the same object as the fine model: less accurate but much faster to evaluate. Space mapping assumes that the family of surrogate models is constructed from the coarse model in such a way that the misalignment between $R_{s}^{(i)}$ and the fine model is minimized. Let $\bar{R}_{s}: X_{s} \rightarrow R^{m}$ be a generic space mapping surrogate model which is the coarse model composed with some suitable space mapping transformations, where $X_{s} \subseteq X_{c} \times X_{p}$, with $X_{p}$ being 
the parameter space of these transformations. At iteration $i$, $i=0,1,2, \ldots$ the surrogate model $R_{s}^{(i)}$ is defined as

$$
R_{s}^{(i)}(\boldsymbol{x})=\bar{R}_{s}\left(\boldsymbol{x}, p^{(i)}\right)
$$

where

$$
p^{(i)}=\arg \min _{p} \sum_{k=0}^{i} w_{i, k}\left\|R_{f}\left(\boldsymbol{x}^{(k)}\right)-\bar{R}_{s}\left(\boldsymbol{x}^{(k)}, \boldsymbol{p}\right)\right\|
$$

and $w_{i, k}$ are weighting factors. We typically use $w_{i, k}=1$ for all values of $i$ and $k$. A variety of space mapping surrogate models is available [1]-[4]. For example, consider input SM [4], in which space mapping is an affine transformation of the coarse model domain of the form $x \rightarrow B x+c$. In this case the generic SM surrogate model takes the form $\bar{R}_{s}(\boldsymbol{x}, p)=\bar{R}_{s}(\boldsymbol{x}, B, c)=R_{c}(B \cdot \boldsymbol{x}+\boldsymbol{c})$.

\section{AsSESSMENT METHOD BASED ON MATCHING CAPABILITY OF SURROGATE MODEL}

In this section we propose an assessment method that measures the capability of a given combination of a coarse model and space mapping surrogate to match the fine model and thus assesses its suitability for space mapping optimization.

Let $X_{T}=\left\{\boldsymbol{x}_{t}^{(1)}, \ldots, \boldsymbol{x}_{t}^{\left({ }^{(}\right)}\right\} \subset X_{f}$ be a set of test points. Let $X_{T . A P P}$ and $X_{T, G E N}$ be subsets of $X_{T}$ such that $X_{T . A P P} \cup X_{T . G E N}=X_{T}$ and $X_{T . A P P} \cap X_{T, G E N}=\varnothing$. Typically, $X_{T . A P P}$ contains about 60 $80 \%$ of the total number of test points. We build a surrogate model $\boldsymbol{R}_{s, t}()=.\overline{\boldsymbol{R}}_{s}\left(., \boldsymbol{p}_{t}\right)$ with $\boldsymbol{p}_{t}$ obtained by parameter extraction, i.e., $\quad p_{t}=\arg \min _{p} \sum_{x \in X_{Y . A P p}}\left\|R_{f}(x)-\bar{R}_{s}(x, p)\right\|$. Note that the parameters of $R_{s . t}$ are determined using fine model data at points in $X_{\text {T.APP }}$. Define $F_{1}$ and $F_{2}$ as

and

$$
F_{1}=\frac{\sum_{y \in X_{Y} A P P}\left\|R_{f}(y)-\bar{R}_{s}\left(y, p^{0}\right)\right\|^{2}}{\sum_{y \in X_{Y}, A P P}\left\|R_{f}(y)-R_{s, t}(y)\right\|^{2}}
$$

$$
F_{2}=\frac{\sum_{y \in X_{r} G Q V}\left\|R_{f}(y)-\bar{R}_{s}\left(y, p^{0}\right)\right\|^{2}}{\sum_{y \in X_{Y} \in G Y}\left\|R_{f}(y)-R_{s t}(y)\right\|^{2}}
$$

where $p^{0}$ denotes initial values of the space mapping parameters.

The first factor, $F_{1}$, measures the approximation capability of the surrogate model, because it is the ratio of the matching error before and after parameter extraction, calculated for the points which were used in parameter extraction. The second factor, $F_{2}$, measures the generalization capability of the model, because it is the ratio of the matching error before and after parameter extraction, calculated for the points which were not used in extraction.

Although both factors are important, $F_{2}$ is the one which tells us more about the suitability of the surrogate model for space mapping optimization. In particular, it allows us to detect over-flexibility of the model (i.e., a situation in which the surrogate model is able to match the fine model perfectly at points used in parameter extraction but exhibits large matching errors for other points). A good surrogate model is characterized by values of $F_{1}$ and $F_{2}$ which are much larger than 1 (although $F_{2}$ is typically considerably smaller than $F_{1}$ ).

In order to perform the assessment, we have to evaluate the fine model at all test points. The recommended number of test points is at least 5 with at least 3 points in $X_{T . A P P}$ and at least 2 in $X_{T . G E N}$. Note, however, that test points can be reused in the actual space mapping optimization. In particular, one can build an initial surrogate model based on all test points and also select the best test point as a starting point for the optimization process. In other words, we can provide a warm start for a space mapping optimization.

Let us consider a seven-section capacitively-loaded impedance transformer [7], an example traditionally used as a benchmark problem for testing space mapping optimization algorithms. The "coarse" and "fine" models for the seven-section impedance transformer are shown in Fig. 1. The design parameters are $\boldsymbol{x}=\left[L_{1} L_{2} L_{3} L_{4} L_{5} L_{6} L_{7}\right]^{T}$. Both models are implemented in Matlab. The design specifications are

$\left|S_{11}\right| \leq 0.07$ for $1.0 \mathrm{GHz} \leq \omega \leq 7.7 \mathrm{GHz}$

Design variables are normalized to the coarse model optimal solution, i.e., the normalized starting point is $x^{(0)}=\left[\begin{array}{lllllll}1 & 1 & 1 & 1 & 1 & 1 & 1\end{array}\right]^{T}$. Characteristic impedances of the coarse model $\boldsymbol{x}_{p}=\left[\begin{array}{lllllll}Z_{1} & Z_{2} & Z_{3} & Z_{4} & Z_{5} & Z_{6} & Z_{7}\end{array}\right]^{T}$ are used as preassigned parameters for implicit space mapping.

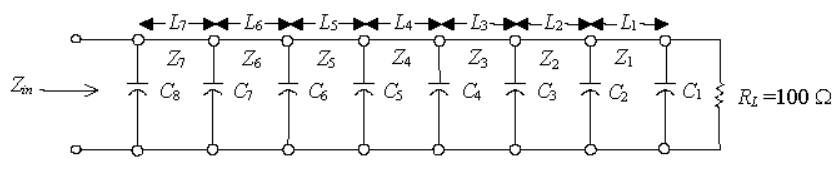

(a)

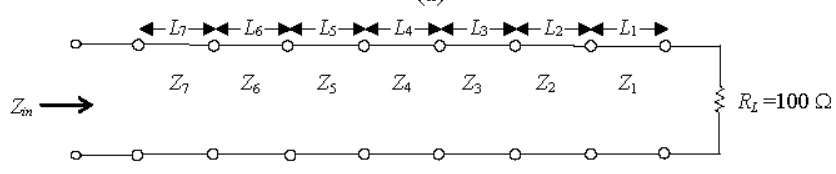

(b)

Fig. 1. Seven-section capacitively-loaded impedance transformer: "fine" model (a) and "coarse" model (b) [7].

We consider the four different surrogate models shown in Table I. Model 1 implements input space mapping, model 2 implements input space mapping and implicit space mapping; model 3 is multiplicative output space mapping, partial input space mapping, while model 4 is model 3 enhanced by implicit space mapping.

We take a test set consisting of $\boldsymbol{x}^{(0)}$ and 5 points randomly selected in the neighborhood of $x^{(0)}$. For each model, we calculate the $F_{1}$ and $F_{2}$ factors and then perform space mapping optimization using all test points to build the initial surrogate model. The values of $F_{1}$ and $F_{2}$ as well as the value of the final specification error obtained after 6 space mapping iterations are shown in Table I.

As we can see from Table I, both approximation and generalization capability is much better for models 3 and 4 than for models 1 and 2. This means that a combination of 
output space mapping and partial input space mapping (with or without implicit space mapping enhancement) is more suitable for a seven-section transformer problem than a regular input space mapping. This is reflected by the values of the specification error: the space mapping algorithm working with models 1 and 2 failed to find a solution satisfying the design specifications. In contrast, the algorithm using models 3 and 4 found solutions which are very close to the fine model optimum $(-0.00987)$.

TABLE I

SURROGATE MODELS, QUALITY FACTORS AND OPTIMIZATION RESULTS FOR SEVEN-SECTION TRANSFORMER EXAMPLE

\begin{tabular}{ccccc}
\hline $\begin{array}{c}\text { Model } \\
\text { No. }\end{array}$ & Surrogate Model & $F_{1}$ & $F_{2}$ & $\begin{array}{c}\text { Final Specification } \\
\text { Error }^{*}\end{array}$ \\
\hline 1 & $\boldsymbol{R}_{c}(\boldsymbol{B} \cdot \boldsymbol{x}+\boldsymbol{c})$ & 3.7 & 1.4 & 0.00684 \\
2 & $\boldsymbol{R}_{c}\left(\boldsymbol{B} \cdot \boldsymbol{x}+\boldsymbol{c}, \boldsymbol{x}_{p}\right)$ & 4.4 & 1.7 & 0.00450 \\
3 & $\boldsymbol{A} \cdot \boldsymbol{R}_{c}(\boldsymbol{x}+\boldsymbol{c})$ & 14.6 & 6.6 & -0.00906 \\
4 & $\boldsymbol{A} \cdot \boldsymbol{R}_{c}\left(\boldsymbol{x}+\boldsymbol{c}, \boldsymbol{x}_{p}\right)$ & 27.2 & 11.7 & -0.00939 \\
\hline \hline
\end{tabular}

${ }^{{ }^{*} \text { Specification error value after } 6 \text { iterations of the space mapping optimization }}$ algorithm

\section{ASSESSMENT METHOD BASED ON CONTRACTION PROPERTY OF SURROGATE MODEL}

The second assessment method is based on estimating the "contraction" property of a given surrogate model and allows us to predict whether the SM algorithm working with this particular combination is likely to converge.

Again, let $X_{T}=\left\{\boldsymbol{x}_{t}^{(1)}, \ldots, \boldsymbol{x}_{t}^{(K)}\right\} \subset X_{f}$ be a set of test points. For each test point $x_{t}^{(j)}$ we build a surrogate model $R_{s . t}^{(j)}()=.\bar{R}_{s}\left(., p_{t}^{(j)}\right)$ with $p_{t}^{(j)}$ obtained using the parameter extraction, i.e., $p_{t}^{(j)}=\arg \min _{p} \sum_{k=1}^{K} w_{k}^{(j)}\left\|R_{f}\left(\boldsymbol{x}_{t}^{(k)}\right)-\bar{R}_{s}\left(\boldsymbol{x}_{t}^{(k)}, p\right)\right\|$. The weighting factors $w_{k}^{(j)}$ should correspond to the distribution of weights used in the actual algorithm. We recommend the following values: $w_{j}^{(i)}=1$ for $j=i$, and $w_{j}^{(i)}=\alpha$ for $j \neq i$, with $\alpha$ defined as $\alpha=\beta /(K-\beta(K-1))$, where $\beta=\left(N_{\max } / 2-1\right) /\left(N_{\max } / 2\right)$ with $N_{\max }$ being the expected maximum number of iterations of the SM algorithm. This definition assures us that the relative effect of increasing $w_{j}^{(i)}$ from $\alpha$ to 1 in the parameter extraction is the same as the effect of adding a next iteration point in the middle of the SM algorithm execution.

Having $R_{s t}^{(j)}$ we find optimal solutions $\boldsymbol{y}_{t}^{(j)}$ of our models as $\boldsymbol{y}_{t}^{(j)}=\arg \min _{\boldsymbol{x}} U\left(\boldsymbol{R}_{s t}^{(j)}(\boldsymbol{x})\right)$.

Now we can define a quality factor $C_{1}$ as follows

$$
C_{1}=\max _{i, j \in(1, \ldots, K\}, i \neq j} \frac{\left\|\boldsymbol{y}_{t}^{(i)}-\boldsymbol{y}_{t}^{(j)}\right\|}{\left\|\boldsymbol{x}_{t}^{(i)}-\boldsymbol{x}_{t}^{(j)}\right\|}
$$

The $C_{1}$ factor measures the "contraction" property of the SM surrogate. In particular, if $C_{1}<1$, we can expect that the SM algorithm should be convergent. This is because in actual optimization, the next iteration point is the optimal solution of the current surrogate model, which means that $C_{1}$ gives an estimate for the distance between two subsequent iteration points with respect to the distance between previous points, i.e., $\left\|\boldsymbol{x}^{(i+1)}-\boldsymbol{x}^{(i)}\right\|<C_{1}\left\|\boldsymbol{x}^{(i)}-\boldsymbol{x}^{(i-1)}\right\|$. Thus, if $C_{1}<1$, the sequence $\left\{\boldsymbol{x}^{(i)}\right\}$ produced by the SM algorithm is a Cauchy sequence and it is convergent if the design space is a closed subset of $R^{n}$. On the other hand, if $C_{1}>1$ then convergence of the algorithm is questionable.

It should be noted, however, that $C_{1}$ is obtained using a few test points, so it is only an estimate of the actual contraction properties of the surrogate model. Therefore, we can conclude that the SM algorithm is likely to converge if $C_{1}$ is clearly smaller than 1 (e.g., $C_{1}<0.7$ ). For values close to 1 , it is difficult to say anything about convergence.

The value of the $C_{1}$ factor can also be used for comparing different SM surrogate models and choosing the best surrogate model for a given optimization problem.

Similarly as for the assessment method described in Section III, we can reuse the test points $\boldsymbol{x}_{t}^{(1)}, \ldots, \boldsymbol{x}_{t}^{(K)}$ in the actual space mapping optimization to provide a warm start for a space mapping optimization by constructing an initial surrogate model which is based on all test points.

Let us consider the the microstrip band-pass filter [8] shown in Fig. 2. The design parameters are $\boldsymbol{x}=\left[\begin{array}{lllll}L_{1} & L_{2} & L_{3} & L_{4} & g\end{array}\right]^{T}$. The fine model is simulated in FEKO [9]. The design specifications are

$\left|S_{21}\right| \leq-20 \mathrm{~dB}$ for $4.5 \mathrm{GHz} \leq \omega \leq 4.7 \mathrm{GHz}$

$\left|S_{21}\right| \geq-3 \mathrm{~dB}$ for $4.9 \mathrm{GHz} \leq \omega \leq 5.1 \mathrm{GHz}$

$\left|S_{21}\right| \leq-20 \mathrm{~dB}$ for $5.3 \mathrm{GHz} \leq \omega \leq 5.5 \mathrm{GHz}$

We consider two coarse models. The first coarse model $R_{c 1}$ is the circuit model implemented in Agilent ADS [10] (Fig. 3). The substrate thickness and relative permittivity for all microstrip elements MLIN, MTEE and MGAP are $0.66 \mathrm{~mm}$ and 9 , respectively. The second coarse model $R_{c 2}$ is the same equivalent circuit, however substrate thickness and permittivity have been tuned for all microstrip elements individually in order to obtain better matching with the fine model. That is why, we know beforehand that model $R_{c 1}$ is better than model $R_{c 2}$.

The initial design is the coarse model $R_{c 1}$ optimal solution $\boldsymbol{x}^{(0)}=\left[\begin{array}{lllll}6.784 & 4.890 & 6.256 & 5.28 & 0.0956\end{array}\right]^{T} \mathrm{~mm}$.

We consider four surrogate models being different combinations of the coarse model and space mapping type shown in Table II. Model 1 combines coarse model $R_{c 1}$ and input space mapping, model 2 uses coarse model $R_{c 1}$ and multiplicative output space mapping. Model 3 and 4 combine coarse model $R_{c 2}$ with input and output space mapping, respectively. Additionally, all models are enhanced by additive output space mapping (term $d$ ).

We take a test set consisting of $x^{(0)}$ and 5 points randomly selected in the neighborhood of $x^{(0)}$. For each model, we determine $C_{1}$ and then perform space mapping optimization. The values of $C_{1}$ and the value of the final specification error after 8 space mapping iterations are shown in Table II. 


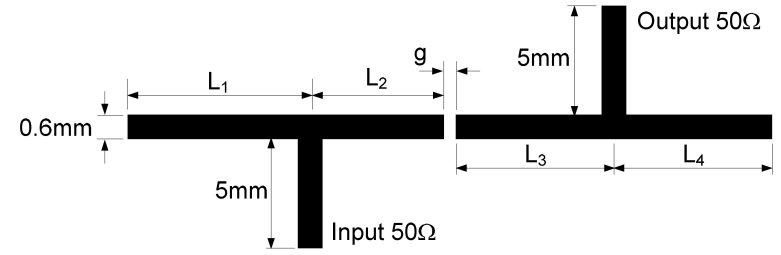

Fig. 2. Geometry of the microstrip band-pass filter [8].

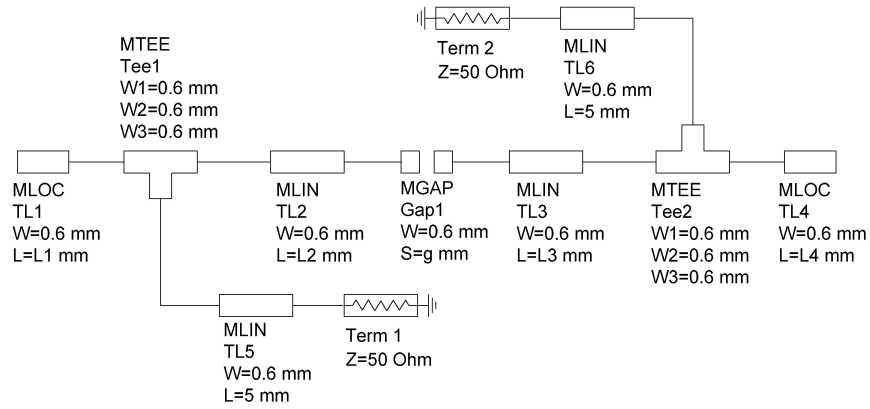

Fig. 3. Coarse model of microstrip band-pass filter (Agilent ADS).

TABLE II

SURROGATE MODELS, QUALITY FACTORS AND OPTIMIZATION RESULTS FOR MICROSTRIP BAND-PASS FILTER EXAMPLE

\begin{tabular}{cccc}
\hline \hline $\begin{array}{c}\text { Model } \\
\text { No. }\end{array}$ & Surrogate Model & $C_{1}$ & $\begin{array}{c}\text { Final Specification } \\
\text { Error }\end{array}{ }^{*}[\mathrm{~dB}]$ \\
\hline 1 & $\boldsymbol{R}_{c 1}(\boldsymbol{B} \cdot \boldsymbol{x}+\boldsymbol{c})+\boldsymbol{d}$ & 1.71 & 0.13 \\
2 & $\boldsymbol{A} \cdot \boldsymbol{R}_{c 1}(\boldsymbol{x})+\boldsymbol{d}$ & 2.86 & 8.62 \\
3 & $\boldsymbol{R}_{c 2}(\boldsymbol{B} \cdot \boldsymbol{x}+\boldsymbol{c})+\boldsymbol{d}$ & 0.79 & -1.23 \\
4 & $\boldsymbol{A} \cdot \boldsymbol{R}_{c 2}(\boldsymbol{x})+\boldsymbol{d}$ & 0.56 & -1.21 \\
\hline \hline
\end{tabular}

$\overline{{ }^{*} \text { Specification error value after } 8 \text { iterations of the space mapping optimization }}$ algorithm.

Convergence properties of the space mapping algorithm using surrogate models 1-4 are shown in Fig. 4.

As follows from Table II, the values of the $C_{1}$ factor for the surrogate models based on the coarse model $\boldsymbol{R}_{c 1}$ indicate potential convergence problems, which are actually the case: the solutions obtained for both models do not satisfy design specifications and the algorithms do not converge as shown in Fig. 4. On the other hand, the values of $C_{1}$ for models based on $\boldsymbol{R}_{c 2}$ are both smaller than 1. This indicates that the contraction properties of surrogate models 3 and 4 are good enough to ensure convergence of the space mapping algorithm. Indeed, the plots shown in Fig. 4 confirm good convergence properties, especially for the algorithm using model 4 (which exhibits the smallest value of $C_{1}$ ). Moreover, the final solutions satisfy the design specifications.

\section{CONCLUSION}

Coarse and surrogate model assessment methods are presented, which allow us to predict the performance of a given combination of a coarse model and space mapping type in the space mapping algorithm before carrying out the actual optimization. The methods can be used to select the coarse model and space mapping type which are best for a given optimization problem. This reduces computational cost and improves performance of the optimization process. Examples confirm the usefulness of our approach.

\section{REFERENCES}

[1] J.W. Bandler, Q.S. Cheng, S.A. Dakroury, A.S. Mohamed, M.H. Bakr, K. Madsen, and J. Sondergaard, "Space mapping: the state of the art," IEEE Trans. Microwave Theory Tech., vol. 52, no. 1, pp. 337-361, Jan. 2004.

[2] J.W. Bandler, Q.S. Cheng, N.K. Nikolova, and M.A. Ismail, "Implicit space mapping optimization exploiting preassigned parameters," IEEE Trans. Microwave Theory Tech., vol. 52, no. 1, pp. 378-385, Jan. 2004.

[3] J.W. Bandler, Q.S. Cheng, D.H. Gebre-Mariam, K. Madsen, F. Pedersen, and J. Sondergaard, "EM-based surrogate modeling and design exploiting implicit, frequency, and output space mappings," IEEE MTT-S Int. Microwave Symp. Digest, Philadelphia, PA, June 2003, pp. 1003-1006.

[4] J.W. Bandler, S. Koziel, and K. Madsen, "Space mapping for engineering optimization," SIAG/Optimization Views-and-News Special Issue on Surrogate/Derivative-free Optimization, vol. 17, no. 1, pp. 1926, 2006.

[5] S. Koziel, J.W. Bandler, and K. Madsen, "A space mapping framework for engineering optimization: theory and implementation," IEEE Trans. Microwave Theory Tech., vol. 54, no. 10, pp. 3721-3730, Oct. 2006.

[6] D. Echeverria, D. Lahaye, L. Encica, E.A. Lomonova, P.W. Hemker, and A.J.A. Vandenput, "Manifold-mapping optimization applied to linear actuator design," IEEE Trans. Magn., vol. 42, no. 4, pp. 11831186, Apr. 2006

[7] M.H. Bakr, J.W. Bandler, K. Madsen, and J. Søndergaard, "An introduction to the space mapping technique," Optimization Eng., vol. 2, no. 4, pp. 369-384, Dec. 2001.

[8] A. Hennings, E. Semouchkina, A. Baker, and G. Semouchkin, "Design optimization and implementation of bandpass filters with normally fed microstrip resonators loaded by high-permittivity dielectric," IEEE Trans. Microwave Theory Tech., vol. 54, no. 3, pp. 1253-1261, March 2006.

[9] FEKO $^{\circledR}$ User's Manual, Suite 4.2, June 2004, EM Software \& SystemsS.A. (Pty) Ltd, 32 Techno Lane, Technopark, Stellenbosch, 7600, South Africa.

[10] Agilent ADS, Version 2003C, Agilent Technologies, 1400 Fountaingrove Parkway, Santa Rosa, CA 95403-1799, 2003.

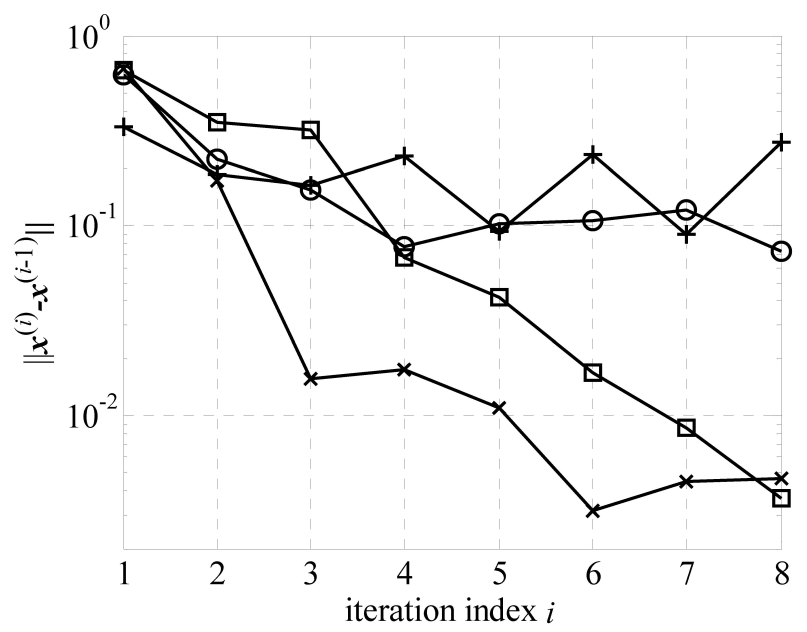

Fig. 4. Microstrip band-pass filter example: convergence properties for the space mapping algorithm using model $1(\mathrm{o})$, model $2(+)$, model $3(\times)$, and model 4 ( $\square$ ). 